\title{
Scale-Space Representation of Lung HRCT Images for Diffuse Lung Disease Classification
}

\author{
Kiet T. Vo and Arcot Sowmya \\ The University of New South Wales \\ Sydney, Australia \\ $\left\{k t v\right.$, sowmya $\varrho_{c}$ se. unsw.edu .au
}

\begin{abstract}
A scale-space representation based on the Gaussian kernel filter and Gaussian derivatives filter is employed to describe HRCT lung image textures for classifying four diffuse lung disease patterns: normal, emphysema, ground glass opacity (GGO) and honey-combing. The mean, standard deviation, skew and kurtosis along with the Haralick measures of the filtered ROIs are computed as texture features. Support vector machines (SVMs) are used to evaluate the performance of the feature extraction scheme. The method is tested on a collection of 89 slices from 38 patients, each slice of size $512 \times 512,16$ bits/pixel in DICOM format. The dataset contains 70,000 ROIs from slices already marked by experienced radiologists. We employ this technique at different scales and different directions for diffuse lung disease classification. The technique presented here has best overall sensitivity of $84.6 \%$ and specificity of $92.3 \%$.
\end{abstract}

Keywords: HRCT diffuse lung disease, texture classification, scale-space feature extraction.

\section{Introduction}

High Resolution Computed Tomography (HRCT) is generally considered to be the best imaging modality for assessment of lung parenchyma in patients likely to have diffuse lung diseases [1]. However, the diagnosis of diffuse lung disease from HRCT images is a difficult task for radiologists because of the complexity and variation in the visual disease patterns on the images. Therefore, the construction of a computeraided diagnosis system for diffuse lung disease is important in providing the radiologist with a "second opinion".

Texture classification has been a significant research topic in image processing, particularly in medical image analysis, and many features have been proposed to represent a texture [2]. The method chosen for feature extraction is clearly critical to the success of texture classification. Five major categories of features for texture identification have been proposed: statistical, geometrical, structural, model-based, and signal processing features. Among these methods, the signal processing approach has advantages in the characterization of the directional and scale features of textures.

In the literature on HRCT lung disease classification, typical methods used are based on histogram statistics, co-occurrence matrices, run-length parameters and 
fractal features such as Uppaluri et al. [3], Chabat et al. [4] and Uchiyama et al. [5]. However, in the recent decade, the feature extraction method based on the wavelet transform has attracted more attention [6-8] with some works applying wavelet transform for lung tissue classification: Shamsheyeva et al. [9] with quincunx wavelet transform (QWF) combined with gray-level histogram features, Shojaii's [10] utilizing the vertical sub-image to extract honeycombing regions, Tolouee et al. [11] with discrete wavelet frames (DWF) and rotated wavelet frames (RWF) to describe features, and experiments conducted by Depeursinge's group using QWF combined with gray-level features, another feature called air-pix (number of air pixels in each ROI) and a set of clinical features [12-14].

However, these wavelet-based methods suffer from the lack of directional information - a unique and important feature of multi-dimensional signals. Therefore, contourlet transform proposed by Do [15] with intrinsic multi-dimensional information (with different number of directions at each scale) and even less computational complexity has recently received increasing attention.

In addition, another approach for signal-processing feature extraction is based on scale-space theory $[16,17]$. Scale space theory is a natural framework to construct multi-dimensional multi-scale textures by deploying multi-scale Gaussian derivative filters up to a certain order. Ginneken [18] applied this method to construct a feature vector in detecting abnormalities in chest radiograph.

In this paper, we apply the scale-space representation of lung HRCT images at different scales and different directions of Gaussian derivative filters to tackle the problem of classification of four diffuse lung disease patterns: normal, emphysema, ground glass opacity (GGO) and honey-combing (HC) (Fig. 1). Bart ter Haa Romeny's group introduced scale-space theory to describe textures, especially in applications of medical issues $[18,19]$ and the most challenge in applying scale-space theory for classification of diffuse lung diseases based on HRCT lung images is to identify the extent of the scale which is appropriate to ensure both high accuracy and low computation time. The second goal of this paper is to evaluate ways to model the outputs of filters after deploying Gaussian derivatives at different scales and directions. In this paper, we use four basic features: mean, standard deviation, skew and kurtosis, along with the Haralick features. At the classification phase, support vector machines (SVMs) are used to evaluate the performance of the feature extraction scheme. The methodology is described in detail in the next section.

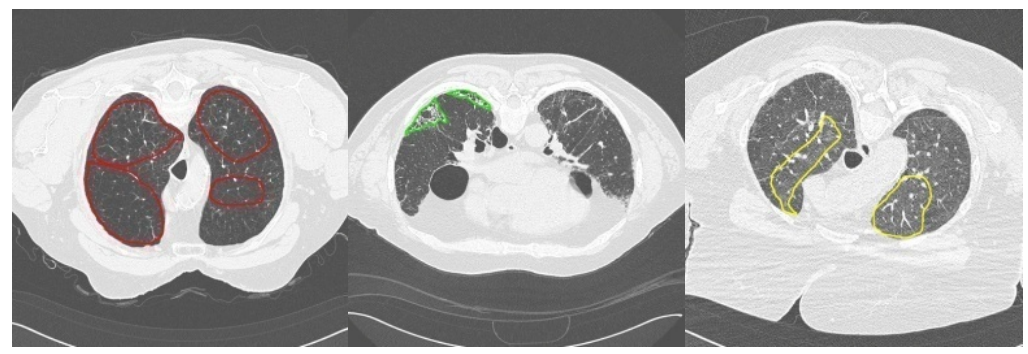

Fig. 1. (a) Emphysema (red) (b) Honeycombing (green) (c) GGO (yellow) 


\section{Methodology}

A texture classification system typically consists of several stages such as image preprocessing, feature extraction and selection, and classification. Each stage is explained below in the context of scale-space texture classification.

\subsection{Scale-Space theory}

A brief description of the scale-space theory is described in [13] and the original textbook [14] may be referenced for more details.

A linear scale-space is a stack of Gaussian blurred images. The generating equation of the images $L$ at different scales is the linear isotropic diffusion equation as given below:

$$
\Delta_{\mathbf{x}} L(\mathbf{x} ; \sigma)=\frac{\partial L(\mathbf{x} ; \sigma)}{\partial t}
$$

where $\Delta$ is the Laplacian, $t=\sigma^{2} / 2$ is the variance, and $\sigma$ iis the scale. Diffusion of intensity is equivalent to convolving the image with Gaussian kernels of variance $\sigma^{2}$ igiven below:

$$
G_{2 D}(\mathbf{x} ; \sigma)=\frac{1}{2 \pi \sigma^{2}} e^{-\frac{\mathbf{x}^{2}}{2 \sigma^{2}}}
$$

The convolution of $L$ with $G_{2 D}$ blurs the image and depending on the scale of the Gaussian kernel will emphasize coarser structures in the image, which is desirable in texture analysis as textures are scale dependent.

Derivatives provide additional information about textures. Derivatives of discrete data are ill-posed. According to scale-space theory, observed data is a Gaussian convolution with the original image:

$$
L(\mathbf{x} ; \sigma)=L(\mathbf{x}) \otimes G_{2 D}(\mathbf{x} ; \sigma)
$$

where $\otimes$ is the convolution operator. If we take the derivative of both sides we obtain:

$$
\frac{\partial}{\partial \mathbf{x}} L(\mathbf{x} ; \sigma)=\frac{\partial}{\partial \mathbf{x}}\left[L(\mathbf{x}) \otimes G_{2 D}(\mathbf{x} ; \sigma)\right]
$$

Both convolution and differentiation are linear operators, so we can exchange the order of these two operators:

$$
\frac{\partial}{\partial \mathbf{x}} L(\mathbf{x} ; \sigma)=L(\mathbf{x}) \otimes \frac{\partial}{\partial \mathbf{x}}\left[G_{2 D}(\mathbf{x} ; \sigma)\right]
$$

which means that the derivative of an observed image can be calculated by convolving the image with the derivative of the Gaussian kernel. The order of the 
derivative determines the type of structure extracted from the image, e.g. the first order derivative emphasizes the edges, the second order emphasizes ridges and corners and so on.

To construct multi-scale texture images we simply convolve the Gaussian derivatives including the zeroth order derivative (the Gaussian kernel itself) with the texture image. Moreover, the $\mathrm{n}^{\text {th }}$ order derivative at any given orientation can be constructed from $\mathrm{n}+1$ independent orientations. For example, if $\mathrm{n}=1$, from only $L_{\mathrm{x}}$ and $L_{\mathrm{y}}$ the derivatives in all other orientations can be calculated using:

$$
L_{\mathbf{x}}^{\theta}(x ; y)=\cos (\theta) L_{\mathbf{x}}(x ; y)+\sin (\theta) L_{\mathbf{y}}(x ; y)
$$

where $L_{\mathbf{x}}^{\theta}$ indicates the derivative of image $L(\mathbf{x})$ in orientation $\theta$.

\subsection{Feature Vector Construction}

Once the medical images are preprocessed, Gaussian derivatives filters (including zeroth order) are used for feature extraction of texture information at different scales and different directions. Mean, standard deviation, skew and kurtosis texture descriptors are then extracted from each filtered ROI. This yields four texture descriptors for each combination of scale and direction. In addition to this, cooccurrence matrices are also calculated at each scale. A co-occurrence matrix captures the spatial dependence of contrast values, depending on different directions and distances specified. Four co-occurrence matrices are calculated for each filtered matrix at each scale; a co-occurrence matrix is calculated for four directions, 0, 45, 90, and 135 degrees at a set distance of one. The following fourteen Haralick texture descriptors are then extracted from each co-occurrence matrix: angular second moment, contrast, correlation, sum of squares (variance), sum average, sum variance, entropy, sum entropy, difference variance, difference entropy, inverse difference moment, information measures of correlation and maximal correlation coefficient [20]. For fast calculation of Haralick texture features, we use the recently-proposed method [21] that uses a recursive blocking algorithm, unrolling and scalar replacement to optimize the code. This smart approach increased the overall performance of the calculation by a factor of 2 .

The final texture descriptor vector had 60 elements per scale per direction. Feature reduction is necessary to reduce the feature space. This can be done by methods of feature selection. However, in this paper, for fast and simple procedure, the size of the texture description vector was reduced to 18 by averaging over the four co-occurrence directions. Through experiments in section 3, the performance of four basic measures (mean, standard deviation, skew and kurtosis) and Haralick measures are evaluated.

\subsection{Classifiers}

The support vector machine (SVM) has exploded in popularity within the machine learning literature and more recently has received increasing attention from the statistics community as well, and SVM has proved to be the best classifier in lung 
disease categorization [22]. The SVM paradigm was originally designed for the binary (two-class) classification problem [23]. To extend the original SVM to multiclass classification, popular methods include one-versus-all method using winnertakes-all strategy; one-versus-one method implemented by max-wins voting; and pair-wise coupling method (the extension of one-versus-one). The strategies are competitive to each other and there is no clear superiority of one method over others [24]. In our experiments, we use SVM with Gaussian radial basis function kernel, quadratic programming method for optimization and one-versus-all method for classification of different diffuse lung disease patterns because this configuration has proven to be the most appropriate in experiments.

\section{Experiments and Results}

The dataset is constructed from a collection of 89 HRCT slices from 38 patients which have been previously investigated and labeled by experienced radiologists, each slice of size 512x512, 16 bits/pixel in DICOM format. From these labeled slices, 73,000 ROIs of size $32 \times 32$ are extracted. The size of ROIs is chosen to maintain the classification sensitivity with the smallest area. Distributions of the ROIs are detailed in Table 1.

Table 1. Distribution of ROIs over lung disease patterns

\begin{tabular}{lcccc}
\hline & Emphysema & GGO & Honeycombing & Normal \\
\hline \# of ROIs & 20,000 & 18,000 & 15,000 & 20,000 \\
\hline \# of patients & 15 & 11 & 8 & 10 \\
\hline
\end{tabular}

The performance of the experiment is evaluated through two measures: sensitivity and specificity. Sensitivity measures the accuracy among negative instances, while specificity measures the accuracy among positive instances:

$$
\text { Sensitivity }=\frac{\mathrm{TP}}{\mathrm{TP}+\mathrm{FN}} ; \quad \text { Specificity }=\frac{\mathrm{TN}}{\mathrm{TN}+\mathrm{FP}}
$$

where TP is true positives, FP false positives, TN true negatives and FN false negatives.

K-fold cross validation [25] is carried out to compute the classification sensitivity and specificity. The advantage of $\mathrm{k}$-fold cross validation is that all the examples in the dataset are eventually used for both training and testing. For our size of dataset, 10fold cross validation is appropriate to ensure that the bias is small and the computational time is acceptable. Moreover, in the experiments, multiple-run k-fold (10-run 10-fold) cross validation is also performed to ensure higher replicability and reliability of the results. Each run is executed with different random splits of the data set.

For all experiments, six directional Gaussian derivative filters $L_{0}, L_{1}^{0^{\circ}}, L_{1}^{90^{\circ}}, L_{2}^{0^{\circ}}, L_{2}^{60^{\circ}}, L_{2}^{120^{\circ}}$, at scales $\sigma=1,2,4,8$ and 16 are used where filters are 
Gaussian derivatives $L_{n}^{\theta}(x, y ; \sigma), \sigma$ denotes the scale, $n$ the order of derivative and $\theta$ the direction in which the derivative is computed. Hence, at each scale, the feature vector consists of $6 \times 4=24$ basic parameters and $6 \times 14=84$ Haralick parameters.

For the classifier, the initial configuration of SVMs is set as mentioned in section 2.4 with two parameters: $\operatorname{cost} \mathrm{C}=1000$ and gamma $\gamma=1$.

The results in Table 2 are obtained from applying Gaussian derivatives at different scales with only 24 basic parameters for each scale. The results show that the best overall sensitivity and specificity are obtained when combining features of all scales. However, it is clearly seen that using only scale $\sigma=2$, we also achieve an acceptable and good result as compared to the best result or in the other words, features at scale $\sigma=2$ play the most important role in discriminating between different patterns of diffuse lung diseases.

Table 2. Results without Haralick measures (24 basic parameters at each scale) Best results are highlighted

\begin{tabular}{|c|cc|c|c|c|c|cc|}
\hline \multirow{2}{*}{ Scale $\sigma$} & \multicolumn{2}{|c|}{ Empysema } & \multicolumn{2}{|c|}{ GGO } & \multicolumn{2}{c|}{ HC } & \multicolumn{2}{c|}{ Normal } \\
\cline { 2 - 10 } & Sensitivity & Specificity & Sensitivity & Specificity & Specificity & Specificity & Sensitivity & Specificity \\
\hline 1 & 76.5 & 85.1 & 72.7 & 83.5 & 70.6 & 82.3 & 75.9 & 87.1 \\
\hline 2 & 86.8 & 89.9 & 80.5 & 86.7 & $\mathbf{8 1 . 6}$ & 85.4 & 83.3 & 90.7 \\
\hline 4 & 78.3 & 85.3 & 72.1 & 84.5 & 71.8 & 80.8 & 80.4 & 83.7 \\
\hline 8 & 80.1 & 85.7 & 75.9 & 80.6 & 74.8 & 79.6 & 80.5 & 86.3 \\
\hline 16 & 73.6 & 80.0 & 73.3 & 80.2 & 70.4 & 75.5 & 75.9 & 83.5 \\
\hline $1,2,4$ & 87.2 & 90.5 & 81.1 & 87.5 & 80.8 & $\mathbf{8 5 . 7}$ & 86.5 & $\mathbf{9 1 . 6}$ \\
\hline $1,2,4,8,16$ & $\mathbf{8 8 . 5}$ & $\mathbf{9 2 . 4}$ & $\mathbf{8 3 . 7}$ & $\mathbf{8 7 . 7}$ & 81.5 & 85.5 & $\mathbf{8 7 . 4}$ & 91.5 \\
\hline
\end{tabular}

To evaluate the performance of the additional Haralick features, we conducted the same experiments at only scale $\sigma=2$ because the size of the feature vector could be huge if we combine all the features of all scales. The results are in Table 3. Although the combination of basic measures and Haralick measures leads to slightly better result, the complexity in computation is much higher, which does not seem to warrant their use.

It is also noticed from both tables that the results for ground glass opacity and honeycombing remain relatively low. Actually, lesions such as ground glass opacity result in similar local mean intensity to honeycombing and are therefore unable to be differentiated when subjected to scale-space filters. In fact, these two patterns are still the most challenging for classification of diffuse lung diseases so far. 
Table 3. Results to evaluate the performance of Haralick features at scale $\sigma=2$ Best results are highlighted

\begin{tabular}{|c|c|c|c|c|c|c|c|c|}
\hline \multirow{2}{*}{ Feature Set } & \multicolumn{2}{|c|}{ Empysema } & \multicolumn{2}{c|}{ GGO } & \multicolumn{2}{c|}{ HC } & \multicolumn{2}{c|}{ Normal } \\
\cline { 2 - 9 } & Sensitivity & Specificity & Sensitivity & Specificity & Specificity & Specificity & Sensitivity & Specificity \\
\hline $\begin{array}{c}24 \text { basic } \\
\text { featuress }\end{array}$ & 86.8 & 89.9 & 80.5 & 86.7 & 81.6 & 85.4 & 83.3 & 90.7 \\
\hline $\begin{array}{c}84 \text { Haralick } \\
\text { features }\end{array}$ & 84.1 & 90.5 & 80.9 & 88.2 & 79.4 & 89.1 & 85.5 & 93.8 \\
\hline $\begin{array}{c}108 \\
\text { combined } \\
\text { features }\end{array}$ & 89.5 & 91.7 & 80.8 & 92.5 & 79.7 & 90.1 & 88.4 & 94.7 \\
\hline
\end{tabular}

\section{Conclusion}

Although the overall results obtained is at the level of "acceptable" when compared to the method previously reported by us using wavelet and contourlet features with sensitivity of $93.40 \%$ and specificity of $98.40 \%$ [26, 27], it also proves the important role of scale-space features in classification of diffuse lung diseases, particularly features at scale 2. Further investigation will integrate scale-space features with the method using wavelet and contourlet feartures to obtain a more robust method.

Acknowledgments. The use of lung HRCT images from the LMIK database, School of Computer Science and Engineering, UNSW, is gratefully acknowledged.

\section{References}

1. Webb, W.R., Muller, N.L., Naidich, D.P.: High-Resolution CT of the Lung. Lippincott Williams \& Wilkins, Philadelphia (2001)

2. Materka, A., Strzelecki, M.: Texture Analysis Methods-A Review. Technical University of Lodz, Institute of Electronics, COSTB 11 report, Brussels (1998)

3. Uppaluri, R., Hoffman, E.A., Sonka, M., Hunninghake, G.W., McLennan, G.: Interstitial lung disease: A quantitative study using the adaptive multiple feature method. Am. J. Respir. Crit. Care Med. 159, 519-525 (1999)

4. Chabat, F., Yang, G.-Z., Hansell, D.M.: Obstructive lung diseases: texture classification for differentiation at CT. Radiology 228, 871-877 (2003)

5. Uchiyama, Y., Katsuragawa, S., Abe, H., Shiraishi, J., Li, F., Li, Q., Zhang, C., Suzuki, K., Doi, K.: Quantitative computerized analysis of diffuse lung disease in high-resolution computed tomography. Med. Phys. 30, 2440-2454 (2003) 
6. Mallat, S.G.: A theory for multiresolution signal decomposition: the wavelet representation. IEEE Transactions on Pattern Analysis and Machine Intelligence 11, 674-693 (1989)

7. Unser, M.: Texture classification and segmentation using wavelet frames. IEEE transactions on image processing 4, 1549-1560 (1995)

8. Wouwer, G.V., Scheunders, P., Dyck, D.V.: Statistical texture characterization from discrete wavelet representations. IEEE Trans. Image Processing 8, 592-598 (1999)

9. Shamsheyeva, A., Sowmya, A.: The anisotropic Gaussian kernel for SVM classification of HRCT images of the lung. In: Proc. Intelligent Sensors, Sensor Networks and Information Processing Conference, pp. 439-444 (2004)

10. Shojaii, R., Alirezaie, J., Babyn, P.: Automatic Segmentation of Abnormal Lung Parenchyma Utilizing Wavelet Transform. In: Alirezaie, J. (ed.) Proc. IEEE International Conference on Acoustics, Speech and Signal Processing, ICASSP 2007, vol. 1, pp. 1217-1220 (2007)

11. Tolouee, A., Abrishami-Moghaddam, H., Garnavi, R., Forouzanfar, M., Giti, M.: Texture Analysis in Lung HRCT Images. In: DICTA '08 on Digital Image Computing: Techniques and Applications, pp. 305-311 (2008)

12. Depeursinge, A., Hidki, A., Platon, A., Poletti, P.-A., Unser, M., Muller, H.: Lung Tissue Classification Using Wavelet Frames. In: Proceedings of the 29th Annual International Conference of the IEEE EMBS Cité Internationale (2007)

13. Depeursinge, A., Iavindrasana, J., Cohen, G., Platon, A., Poletti, P.A., Muller, H.: Lung Tissue Classification in HRCT Data Integrating the Clinical Context. In: 21st IEEE International Symposium on CBMS '08, Computer-Based Medical Systemspp. 542-547 (2008)

14. Depeursinge, A., Iavindrasana, J., Hidki, A., Cohen, G., Geissbuhler, A., Platon, A., Poletti, P.-A., Muller, H.: A classification framework for lung tissue categorization. In: SPIE (2008)

15. Do, M.N., Vetterli, M.: The contourlet transform: an efficient directional multiresolution image representation. IEEE Transactions Image on Processing 14, 2091-2106 (2005)

16. Gangeh, M., ter Haar Romeny, B., Eswaran, C.: Scale-Space Texture Classification Using Combined Classifiers, pp. 324-333 (2007)

17. ter Haar Romeny, B.M.: Front-End Vision and Multi-scale Image Analysis: Multi-scale Computer Vision Theory and Applications (Written in Mathematica). Kluwer Academic Publishers, Dordrecht (2003)

18. van Ginneken, B., Katsuragawa, S., ter Haar Romeny, B.M., Doi, K., Viergever, M.A.: Automatic detection of abnormalities in chest radiographs using local texture analysis. IEEE Trans. Med. Imaging 21(2), 139-149 (2002)

19. Gangeh, M.J., Duin, R.P.W., Eswaran, C., Haar Romeny, B.M.: Scale Space Texture Classification Using Combined Classifiers with Application to Ultrasound Tissue Characterization, pp. 287-290 (2007)

20. Haralick, R.M., Dinstein, I., Shanmugan, K.: Textural features for image classification. IEEE Transactions on Systems, Man, and Cybernetics SMC-3, 610-621 (1973)

21. Miyamoto, E., Merryman, T.: Fast calculation of Haralick texture features (2008)

22. Depeursinge, A., Iavindrasana, J., Hidki, A., Cohen, G., Geissbuhler, A., Platon, A., Poletti, P.-A., Muller, H.: A classification framework for lung tissue categorization. In: SPIE, vol. 6919 (2008)

23. Burges, C.J.C.: A Tutorial on Support Vector Machines for Pattern Recognition. Kluwer Academic Publishers, Boston (1998) 
24. Duan, K., Keerthi, S.S.: Which Is the Best Multiclass SVM Method? An Empirical Study: Multiple Classifier Systems, 278-285 (2005)

25. Devijver, P.A., Kittler, J.: Pattern Recognition: A Statistical Approach. Prentice-Hall, London (1982)

26. Vo, K., Sowmya, A.: Directional Multi-scale Modeling of High-Resolution Computed Tomography (HRCT) Lung Images for Diffuse Lung Disease Classification. In: CAIP (2009)

27. Vo, K., Sowmya, A.: Diffuse Lung Disease Classification in HRCT Lung Images Using Generalized Gaussian Density Modeling of Wavelets Coefficients. In: IEEE International Conference on Image Processing, Cairo, Egypt (2009) 